\title{
New Perspectives in Cancer Therapy: The Biotin-Antitumor Molecule Conjugates
}

\author{
Giuseppe Tripodo ${ }^{1 \#}$, Delia Mandracchia ${ }^{2}$, Simona Collina ${ }^{1 *}$, Marta Rui ${ }^{1}$ and Daniela Rossi ${ }^{1 \#}$ \\ ${ }^{1}$ Department of Drug Sciences, University of Pavia, Viale Taramelli 12, 27100 Pavia, Italy \\ ${ }^{2}$ Department of Pharmacy, University of Bari "Aldo Moro", Via Orabona 4, 70125 Bari, Italy \\ \#Giuseppe Tripodo and Daniela Rossi contributed equally to this work
}

\begin{abstract}
Chemotherapy is still the first-line treatment of cancer, even if drugs currently used in therapy generally possess high toxicity and poor selectivity. In the last two decades several efforts have been made to overcome these drawbacks by specifically carrying anticancer drugs to the tumors. Among the different approaches, the so called vitaminmediated drug targeting has recently emerged as a novel and valuable strategy. Indeed, the linkage of cytotoxic drugs to selected vitamins, leading to vitamin-drug conjugates, would result in specifically delivering great amounts of the targeted drug at high doses to cancer cells. Among vitamins, biotin seems to be the most promising targeting agent. The aim of this review is to get an overview on recent success in the conjugation of biotin with molecules endowed with anticancer properties.
\end{abstract}

Keywords: Anticancer drugs; Biotin; Doxorubicin; Drug targeting; Gemcitabine; Taxol

\section{Introduction}

Chemotherapy is still the first-line treatment of cancer, even if the drugs currently used in therapy, generally possess high toxicity and poor selectivity. Drugs commonly used in cancer therapy are characterized by different mechanisms of action i.e. i) toxicity to specific cancer cells, ii) anti-proliferative activity or iii) capability of modifying the cell cycle at specific phases [1-5]. As a direct consequence of the cellular activity of anti-cancer drugs, in particular with respect to rapidly proliferating and dividing cells, they show low selectivity towards not-cancerous cells like red blood cells, gut epithelia, bone marrow or hair follicles [6]. On the other side, due to the slow proliferation typical of certain tumors, several anticancer drugs used so far result ineffective against such tumors, thus leading to the need of high dose therapies to limit the cancer growing. These high doses could destroy part of the cancer cells but also result in a hard damage to the adjacent normal-proliferating cells. Due to these drawbacks, the cancer therapy has to be discontinued and sometimes stopped before the tumor mass has been reduced or eliminated $[7,8]$. Thus, reaching a real selectivity of anti-cancer drugs is a milestone and still a challenge in cancer research. To this aim, different approaches could be applied. One of the most common consists in providing the drug with a suitable carrier. The polymeric drug carriers emerged as the most effective ones because of their versatility of use as well as their straightforward chemical modification [9-17]. These systems usually bring to a passive drug targeting due to their ability to accumulate in a specific organ as a result of their route of elimination or for particular organotropism. A more specific drug targeting could be attained by providing the carrier with specific targeting agent, such as antibodies, vitamins, magnetic particles, hormones or peptides [1824]. Another widely applied approach for drug targeting is the direct linking of the targeting molecule to the drug to form a new chemical entity pharmacologically active per se or a prodrug [7,25-27]. In this context, the so called vitamin-mediated drug targeting has recently emerged as a novel concept in specifically carrying anticancer drugs to the tumors [7,28-31]. As known, for their survival living cells need to consume vitamins during their life cycle. This is particularly true for those cells that rapidly divide, such as cancer cells. Indeed, the intense metabolic activity of cancer cell arises from their fast growth and is accompanied by a strong use of essential vitamins; consequently the receptors involved in vitamin internalization are overexpressed on the cell surface. This concept is essential from a therapeutic point of view. In fact, it has been argued that the linkage of cytotoxic drug to selected vitamins, leading to vitamin-drug conjugates, would result in specifically delivering great amounts of the targeted drug at high doses to cancer cells, and thus, represents an attractive and valuable approach for targeting tumor cells. In this context, biotin, folic acid, vitamin B12 and riboflavin, that are essential for the division of all cells and in particular for tumor cells, have been recently experimented as targeting agents [20,32-35]. Interestingly, among these vitamins, biotin seems to be the most promising targeting agent. It is well known that the uptake of biotin by mammalian cells is receptor-mediated [36]. Among the first insights on cell surface biotin receptors there are the studies from SM Grassl in 1992 and Janos Zempleni and Donald M. Mock in 1998. Studying the biotin uptake by human peripheral blood mononuclear cells (PBMC) Zempleni and Mock found that "...in the presence of ouabain, biotin uptake into PBMC is dependent on normal function of Na-K-ATPase. It seems likely that biotin is co-transported with sodium, because biotin uptake into PBMC was reduced when extracellular sodium was replaced by choline, lithium, or ammonium. Likewise, as observed by others, the biotin uptake into rat hepatocytes, rat intestinal cells, or Hep G2 cells was reduced by ouabain and by sodium-free media." [37-39]. In a subsequent study, they also found that PBMC accumulate biotin by a transporter that is specific for biotin. These studies provided evidence that another, less specific transporter in mammalian cells may bind biotin, pantothenic acid, and similar compounds. However, the contribution of this transporter to biotin uptake into PBMC is quantitatively minor [38]. The main transporter for biotin has been found in the sodium-dependent multivitamin transporter (SMVT), which has been found to be overexpressed in several aggressive cancer lines such as leukemia (L1210FR), ovarian

*Corresponding author: Simona Collina, Department of Drug Sciences, University of Pavia, Viale Taramelli 12, 27100 Pavia, Italy, Tel: +39 (0) 382987379; E-mail: simona.collina@unipv.it

Received March 18, 2014; Accepted April 21, 2014; Published April 23, 2014

Citation: Tripodo G, Mandracchia D, Collina S, Rui M, Rossi D (2014) New Perspectives in Cancer Therapy: The Biotin-Antitumor Molecule Conjugates. Med chem S1: 004 doi:10.4172/2161-0444.S1-004

Copyright: $\odot 2014$ Tripodo G, et al. This is an open-access article distributed under the terms of the Creative Commons Attribution License, which permits unrestricted use, distribution, and reproduction in any medium, provided the original author and source are credited. 
(OV 2008, ID8), colon (Colo-26), mastocytoma (P815), lung (M109), renal (RENCA, RD0995), and breast (4T1, JC, MMT06056) cancer cell lines [40,41]. Additionally, its overexpression was found superior to that of folate receptor [30]. This is not surprising because biotin belongs to a particular category of (exogenous) micronutrients required for cellular functions and, in particular, for cell growth [42,43]. For this reason, the biotin demand in tumors, especially in the rapidly growing tumors, is higher than normal tissues.

One of the first papers dealing with the evaluation of the effectiveness of folate, vitamin B12 or biotin-functionalized polymeric materials as active drug targeting agents to tumor cells was published in 2004 by Russell-Jones et al. Particularly, by examining in vitro several tumor cell lines, they reported "cell types appeared to cluster in three categories. Category one, represented by 0157 , BW5147, B16, LL-2 and HCT-116, were shown to have no enhancement of uptake with any of the targeting agent. Category two, represented by L1210FR, Ov2008, ID8, and Ovcar, showed enhanced uptake of folate and biotintargeted polymers, but not vitamin B12 (Cbl). Category three, tumors represented by Colo-26, P815, M109, RENCA, RD995, 4T1, JC and MMT060562, showed enhanced uptake of vitamin B12 and biotinlabeled polymers" [31]. They also reported that the cells overexpressing the receptors for folate or vitamin $\mathrm{B} 12$, also overexpress receptors for biotin. Furthermore, an in vivo study revealed that a rhodamine-labeled polyacrylate reached relatively high level in the mice tumor cells only when targeted by biotin or folate, but not by vitamin B12. Moreover, although the relative increase in uptake of targeted polymer is varying between different cell lines, as a tendency, the biotin labeled polymer showed the highest level of uptake. The same trend has been observed when the cytotoxic drug methotrexate has been conjugated to the vitamin targeted polymer. Indeed, the biotin targeted systems resulted more cytotoxic than the folate or vitamin B12-targeted ones. They also found that the treatment of Colo-26-bearing mice with vitamindoxorubicin- polymer conjugates resulted in greatly enhanced killing, with respect to the control, when using the biotin targeted drugpolymer complex. However, the same effect was not seen when either folate or vitamin B12 were used as targeting agents [31]. These results are clearly addressing biotin as the most promising targeting agent.

As we will report in this review, the biotin-mediated drug targeting of anti-tumor drugs emerges as an attractive approach to both improve efficiency and efficacy and reduce cytotoxicity of anti-cancer therapy. It is worth noting that the application of biotin-anticancer drug conjugates in drug development is still at its early stage, and translation in clinical research is being done progressively. The purpose of this review is to bring an objective view on the antitumor potential of biotin conjugates showing recent works, with particular emphasis on the last 4 years. A focus will be made on conjugates in which biotin is linked to i) drugs used in cancer therapy or their derivatives, ii) anticancer drug candidates and iii) protein with antitumor effect.

\section{Biotinylation of Drugs used in Cancer Therapy or their Derivatives}

\section{Biotin conjugated to taxoids}

The microtubules, which are formed by $\alpha$ - and $\beta$-tubulin heterodimers, are the main components of the cytoskeleton and possess a variety of functions in intracellular processes, i.e. in maintaining cell shape and structure or in regulating the receptors moving inside the cell [44]. To date, numerous antimicrotubule agents with antimitotic properties and anticancer potential have been discovered and developed $[3,45,46]$. Among these agents, Paclitaxel is a natural highly oxygenated diterpenoid that was first isolated in 1971 from the stem bark of the western yew, Taxus brevifolia. It is widely used to treat a variety of solid tumors such as breast, ovarian, non-small cell lung, head and neck cancers $[47,48]$. Since Paclitaxel was discovered, its structure has been extensively studied and modified yielding the so called taxoids, a class of proven anticancer drugs which promote microtubule assembly and suppress microtubule dynamics, thus causing the block of mitotic activity and subsequent cellular apoptosis $[3,5]$.

In 2010, Chen et al., applied what they called "an efficient mechanism-based tumor-targeting drug delivery system, based on tumor-specific vitamin-receptor mediated endocytosis...”. The Authors prepared a biotin conjugates with one of the new-generation taxoids, SBT-1214 [40]. The approach of this work is clear: to use the biotin- SBT-1214 conjugate 1 (Figure 1), which is characterized by an intracellularly labile disulfide linkage, to target the cancer cell by exploiting the overexpression of biotin receptor on the tumor cell surface.

In order to follow the entire process involved in the tumor targeting of the conjugate, the Authors synthesized three fluorescent biotin conjugates (Figure 2): i) the biotin-fluorescein conjugate 2, to observe the receptor-mediated endocytosis by tracking the fluorescent biotin in its route, ii) the biotin-coumarin conjugate 3 , as a fluorogenic probe to evaluate the intracellular degradation of the disulfide linkage between the linker and coumarin, which becomes fluorescent only when it is released as a free molecule via disulfide cleavage of the spacer and iii) the biotin- SBT-1214 -fluorescein conjugate 4, to validate the whole internalization by receptor-mediated endocytosis and drug release processes, wherein the released fluorescent taxoid should bind to microtubules in the cancer cells.

In details, conjugates 2,3 and 4 have been tested on L1210FR murine leukemia cell line, which overexpress receptors for biotin on their cell surface. Firstly, the Authors proved the internalization of the biotin-fluorescein conjugate 2 into the leukemia cells, by evaluating the intracellular fluorescence after incubation with the probe. Furthermore, they confirmed that the internalization was receptor-mediated by preincubation of the same cells with an excess of free biotin. They found that in these conditions the fluorescence of the biotin-fluorescein conjugate 2 decreased about 4.5 times, thus confirming that the biotin-fluorescein internalization is receptor-mediated. Secondly, the Authors confirmed the effectiveness of the fluorogenic biotin- coumarin conjugate 3 by incubating the chosen cell line with conjugate 3 . Differently from

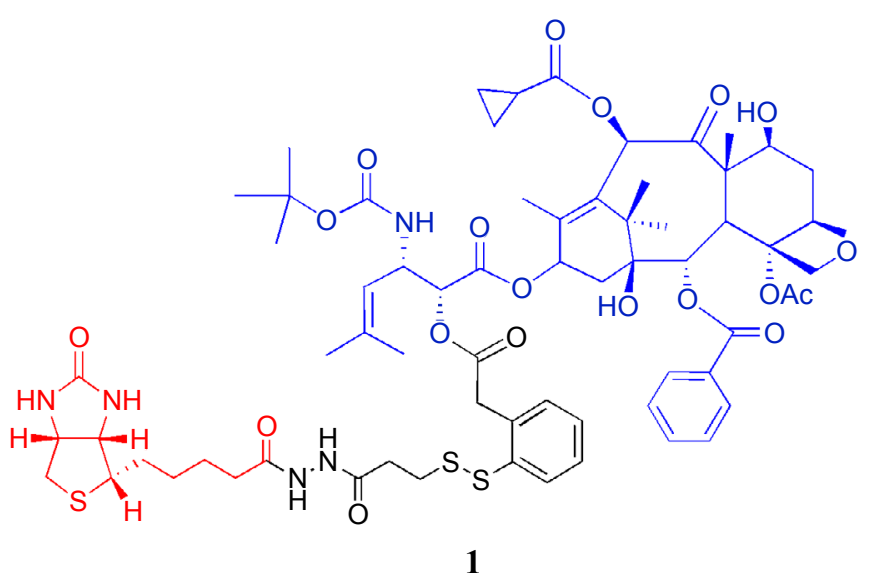

Figure 1: Chemical structure of biotin- SBT-1214 conjugate 1. 


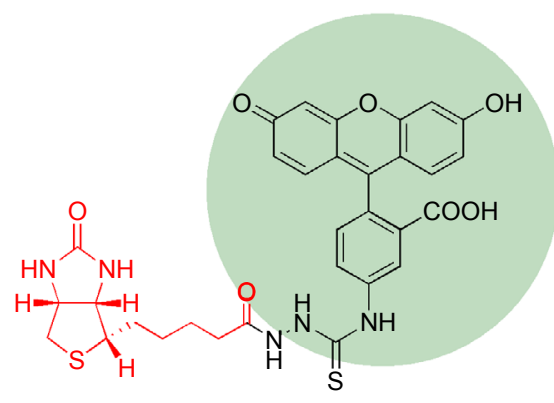

2

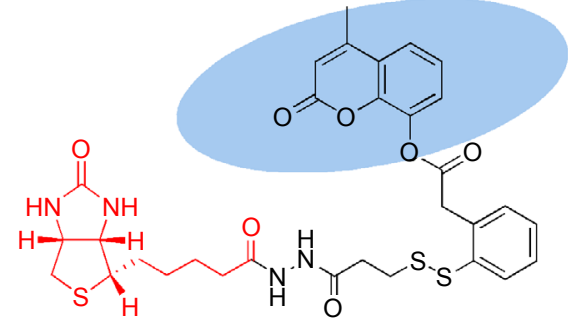

3

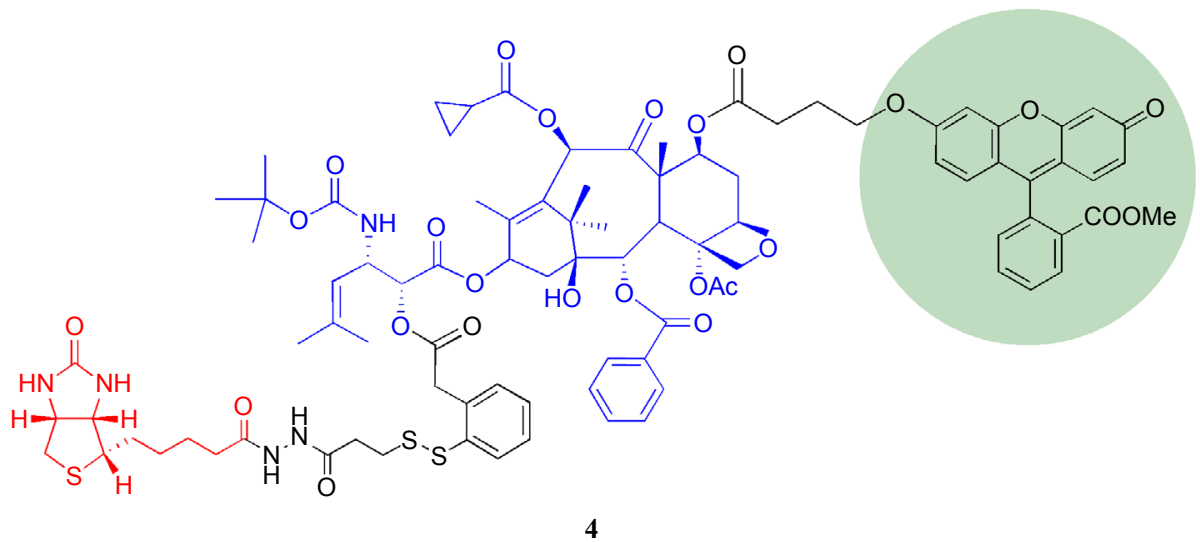

Figure 2: Chemical structure of biotin-fluorescein conjugate 2, biotin-coumarin conjugate 3 and biotin-SBT-1214-fluorescein conjugate 4 .

conjugate 2 , the intracellular fluorescence for conjugate 3 could be only evidenced after reductive cleavage of the spacer. Due to the visualized intracellular fluorescence, this study clearly demonstrated that the intracellular release of coumarin via cleavage of the disulfide linkage by GSH followed by thiolactonization took place as designed. Successively, they tested the biotin-SBT-1214-fluorescein conjugate 4 . These results confirmed the internalization of the conjugate and also clearly proved that the released fluorescent taxoid binds to the microtubules. A crossconfirmation of the previous data has been performed by testing conjugate 4 using two more cell lines, L1210 murine leukemia cell line and WI38 noncancerous human lung fibroblast cell line, which do not overexpress receptors for biotin on their cell surfaces. This experiment resulted in a much stronger (about 12-13 times) fluorescence in L1210FR cells (with biotin receptors overexpressed) as compared to that in L1210 cells or WI38 cells, so confirming the biotin-mediated drug targeting. Finally, the Authors evaluated the cytotoxicity of the compound 1 against L1210FR, L1210 and WI38 cell lines by using the MTT assays. The conjugate 1 resulted high cytotoxic against L1210FR ( IC $_{50} 8.8 \mathrm{nM}$ ), while the cytotoxicity against L1210 ( IC $_{50} 522$ $\mathrm{nM})$ and WI38 ( $\left.\mathrm{IC}_{50} 570 \mathrm{nM}\right)$ cell lines were 59 times and 65 times lower, respectively, as a further confirmation of the receptor mediated endocytosis. A fundamental consideration arises from the last results: the low toxicity that these conjugates showed against not-cancerous cells demonstrates that these systems could differentiate cancer cells from normal ones so reducing the side effects related to the use of cytotoxic drugs. These results are of paramount importance in the field of biotin targeted drugs because clearly demonstrated that: i) the biotin-drug conjugates are preferentially internalized into cancer cells, ii) the toxicity against normal cell is significantly reduced, and iii) the intracellular cleavage of the employed spacer allows a better systemic stability and a lower interaction with non-cancerous cells.

\section{Biotin conjugated to doxorubicin}

The antibiotic doxorubicin is extensively used against different human cancers, such as breast cancer, soft tissue sarcomas, and Hodgkin's and non-Hodgkin's lymphomas. The anticancer activity of doxorubicin has been addressed to several mechanisms of action: i) inhibition of DNA synthesis in the tumor cell, ii) generation of free radicals, iii) DNA adduct formation and DNA cross-linking, iv) interference with DNA strand separation and DNA helicase, v) interaction with cell membranes, vi) induction of DNA damage through interference with topoisomerase II, vii) induction of apoptosis and viii) growth arrest of tumor cells [49]. Nevertheless, the clinical use of doxorubicin is affected by several harmful side effects, among them, the cardiotoxicity is the most important one, leading to cardiomyopathy and congestive heart failure. Indeed, doxorubicin is known to cause cardiotoxicity through multiple routes, including the build-up of reactive oxygen species and disruption of the calcium homeostasis in cardiac myocytes. Several approaches have been proposed to limit its cardiotoxic effects, i.e. incorporation of the drug inside liposomes, formation of prodrugs proteolytically activated in the tumor cells, or the formation of polymeric prodrugs bearing specific drug-targeting moieties. Although these approaches are valuable, they are still not resolving the drawbacks connected to doxorubicin side effects [50]. Aiming at limiting the adverse side effects of doxorubicin, in 2010 Ibsen S. et al. designed and synthesized the biotin-doxorubicin conjugate 5 (Figure 3), in which the amine group of doxorubicin was derivatized with a photocleavable biotinylated spacer [51].

They demonstrated that the active drug is released only after the internalization of the conjugates in cancer cells and the subsequent activation of the photocleavable group via exposure to UV at 350 $\mathrm{nm}$, thus minimizing the cytotoxic effects on not-cancerous cells. 
<smiles>COc1cccc2c1C(=O)c1c(O)c3c(c(O)c1C2=O)C[C@@H](C(=O)OCO)C[C@@H](O[C@@H]1C[C@H](NC(=O)OC(C)c2cc(CNC(=O)CCC(=O)NCCOCCOCCNC(=O)CCCCC4SCC5NC(=O)NC54)ccc2[N+](=O)[O-])C(O)C(C)O1)O3</smiles>

Figure 3: Chemical structure of biotin-doxorubicin conjugate 5

The Authors affirmed that the UV irradiation of deep tissues could be performed by implanting optic fibers or UV light-emitting diodes into the tumor mass and that light with $350 \mathrm{~nm}$ wavelength has significant penetration through tissue that does not contain melanin with minimal absorption also by DNA [52]. The Authors also studied the cell uptake and cellular localization in PTK2 epithelial cells, which are sensitive to doxorubicin and capable to remain flat during cell division, allowing the mitotic spindle and chromosomes to be readily visible. Interestingly, they found that the intracellular localization of doxorubicin was distinct from that of biotin-doxorubicin conjugate. In details, doxorubicin was found into the cell and concentrated almost exclusively in the nucleus, where it was associated with the chromatin most likely through a DNA intercalation mechanism. As a consequence, the nuclear membrane was degraded and doxorubicin strongly localized to the exposed chromosomes. On the contrary, the biotin-doxorubicin conjugate $\mathbf{5}$ showed a quick internalization and did not concentrate in the nucleus, but around it. The biotin-doxorubicin conjugate was thus excluded from the mitotic spindle and the associated chromosomes. Moreover, its intracellular concentration resulted 10 times higher than that of doxorubicin and the cells much healthier, appearing to be undergoing normal mitosis. After one hour of UV exposition, the photocleavage of the spacer allowed the release of the naturally fluorescent doxorubicin, which, in turn, accumulated into the nucleus, as proved by the intense fluorescence observed. The Authors also evaluated the cytotoxicity of both doxorubicin and its conjugate 5 on the human lung cancer cell line A549. The results of the cell proliferation assay clearly evidenced a significantly lower cytotoxicity of conjugate 5 with respect to that of free doxorubicin, being the $\mathrm{IC}_{50}$ value equal to $1.2 \mu \mathrm{M}$ and $250 \mu \mathrm{M}$ for doxorubicin and the biotin-doxorubicin conjugate, respectively. As expected, after UV exposure of the treated cells, the $\mathrm{IC}_{50}$ values for conjugate 5 resulted comparable to that of free doxorubicin. Another important consideration that emerges from this study is that the incorporation of the biotin moiety might help to increase the clearance rate of the freely circulating biotinylated doxorubicin, because it has been demonstrated that the elimination of biotinylated molecules from the human body could be higher than the corresponding notbiotinylated ones [25,53]. Summarizing, this approach allowed to obtain a system able to spare not-cancer cell from the drug cytotoxicity so targeting the conjugate to tumor cells, where the drug could be "activated" by in-situ UV irradiation. Overall, result obtained so far strongly suggest that the biotin-doxorubicin conjugate 5 could be a good drug candidate to reduce the undesirable systemic side effects of doxorubicin.

\section{Biotin conjugated to gemcitabine}

Gemcitabine is an anti-metabolite used to control non-small cell lung, pancreatic, metastatic breast and current ovarian cancers [54,55]. After cellular uptake, gemcitabine is phosphorylated to gemcitabine monophosphate ( $\mathrm{dFdCMP}$ ) which, in turn, is converted to gemcitabine di- and triphosphate (dFdCDP and $\mathrm{dFdCTP}$, respectively) that are the active drug metabolites. The triphosphate analogue of gemcitabine acts i) replacing one cytidine during DNA replication and ii) inhibiting the DNA polymerase [56]. Some characteristic of gemcitabine, i.e. its short plasma half-life (9-13 $\mathrm{min}$ in human) due to its rapid renal clearance, and its myelosuppression side effect, contribute to decrease the gemcitabine chemotherapeutic index [57].

In their efforts to overcome the side effects of gemcitabine, in two recent papers Maiti et al. and Bhuniya et al. synthesized two new theranostic anticancer targeted prodrugs of gemcitabine (conjugates 6 and 7 , Figure 4 ), which are characterized by a disulfide cleavable linker containing a fluorescent probe (coumarin for 6 and IR fluorescent BODIPY fluorophore for 7) [55,58]. A theranostic is a special type of Drug Delivery System (DDS) useful for therapeutic and diagnostic applications, which is able to provide not only a specific cellular drug release, but also a real time monitoring of the drug released in tissue [59].

The Authors studied the behavior of 6 and 7 in the presence of glutathione (GSH), which is the most abundant thiol in cells, or dithiothreitol (DTT) and demonstrated that gemcitabine release is due to cleavage of the sulfide bond upon GSH or DTT treatment,

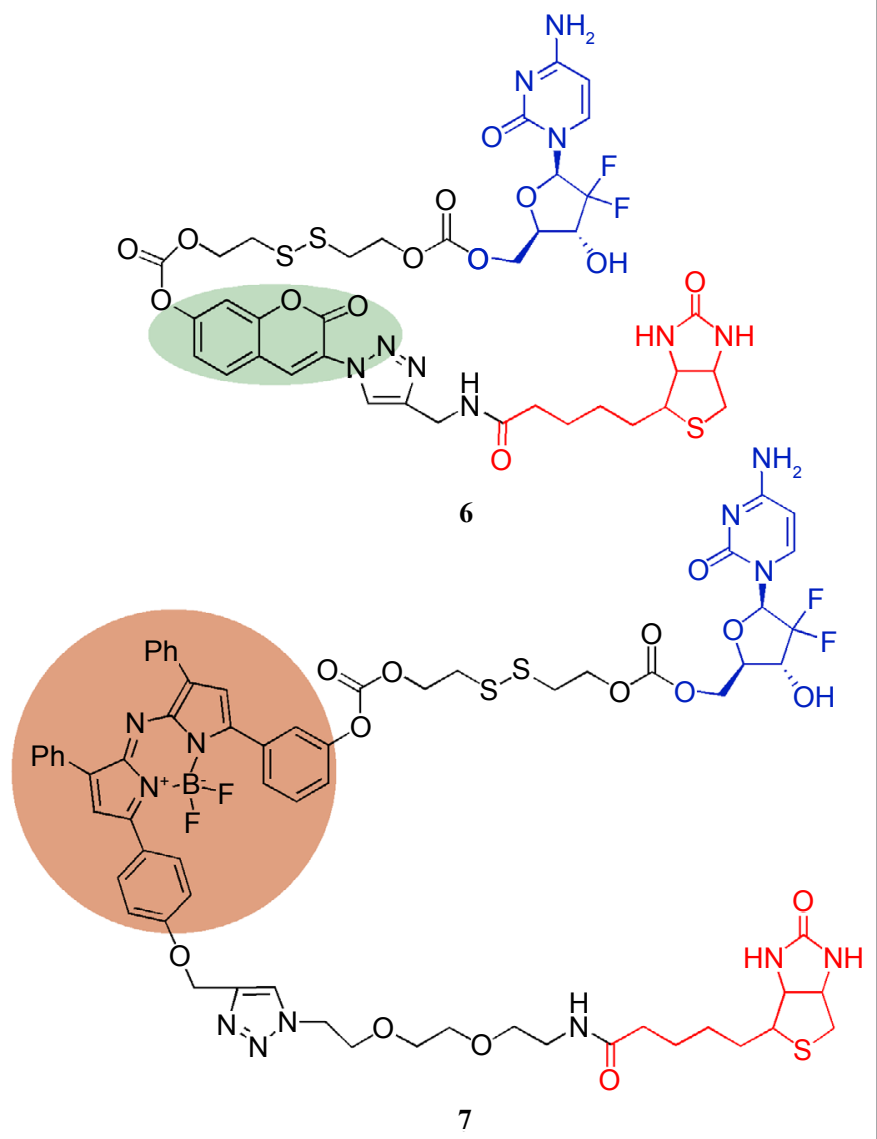

Figure 4: Chemical structure of biotin-gemcitabine conjugates 6 and 7. 
followed by an intramolecular nucleophilic substitution of the thiol at the carbamate moiety. This mechanism of gemcitabine release is supported by further studies carried out in the presence of different biologically relevant analyte containing or not thiol groups, such as cysteine, homocysteine, non-thiol amino acids or metal ions. Results clearly evidenced that conjugates 6 and 7 undergo only a thiol-mediate cleavage, with no significant interference from other molecules present in biological environment. To investigated whether the biotin moiety can guide 6 and 7 to biotin receptor-positive or biotin receptor-negative tumor cells, Authors evaluated the fluorescence by confocal microscopy in A549 (biotin receptor-positive) and WI38 (biotin receptor-negative) cells pre-incubated with 6 and 7. A strong fluorescence intensity was only observed in the A549 cells, thus confirming that biotin acts as targeting agent to cancer cells. Additionally, to investigate the intracellular location of gemcitabine release, Authors performed colocalization experiments using fluorescent endoplasmic reticulum (ER) and lysosome-selective markers. They found that biotin-gemcitabine conjugate 7 , containing the near IR BODIPY fluorophore, localized to the ER, while biotin-gemcitabine conjugate 6 , containing coumarin, localized to the lysosome, probably by receptor mediated endocytosis. The subsequent thiol-induced disulfide cleavage released gemcitabine which, in turn, diffused to the cell nucleus. Finally, MTT assay performed on A549 cells clearly evidenced a stronger anticancer effect for the biotin conjugates 6 and 7 with respect to their analogues without the biotin moiety, further confirming the biotin-mediated targeting to tumor cells. In summary, also in these studies the conjugation of anticancer drugs with biotin emerged as a successful approach to spare cancer and non-cancer cells, thus increasing the cell specificity of the drug.

\section{Biotinylaton of Potential Anti-tumor Drug Candidates}

\section{Biotin conjugated to Annonaceous Acetogenis derivatives}

Squamocin (compound 8, Figure 5) and bullatacin (compound 9, Figure 5) are annonaceous acetogenins (ACGs) belonging to adjacent bis-THF type of acetogenins $[41,60,61]$. ACGs are secondary metabolites occurring in some plants of Annonaceae family. They are derivatives of long-chain fatty acid (C32 or C34) bearing a terminal $\gamma$-lactone ring and have been reported to potently inhibit the activity of NADH-ubiquinone oxidoreductase (respiratory complex I). These long-chain fatty acid derivatives display impressive cytotoxicity against various tumor cell lines $\left(\mathrm{IC}_{50}\right.$ value ranging from $10^{-6} \mathrm{M}$ to $10^{-14} \mathrm{M}$ ) as well as in vivo antitumor effect [41,62-64].

In a just-published research paper, Shi et al. report on the synthesis of nineteen biotinylated derivatives of 8 and 9 as well as on their cytotoxicity against three cancer cell lines: 4T1 (breast cancer), P815 (mastocytoma) and L1210 (leukemia). The first two cell lines overexpress the receptor for biotin, while the third one is not overexpressing the receptor [41]. Generally, they found that the cytotoxic activity of the biotin- ACG conjugates against L1210 cell line is almost the same as that of the parent 8 and 9 . This datum is fundamental to asses that biotinylation of 8 and 9 does not adversely affect their cytotoxic potential. On the other side, the biotin- ACG conjugates showed significantly higher cytotoxicity than 8 and 9 against $4 \mathrm{~T} 1$ and P815 cell lines. In particular, the biotin- squamocin conjugate 10 (Figure 6) resulted ten and twenty six times more active than 8 against $4 \mathrm{~T} 1$ and P815 cells, respectively. Similarly, the biotinbullatacin conjugate 11 (Figure 6), resulted three and eight times more potent than 9 against 4T1 and P815 cells, respectively. Taken together, the results clearly demonstrate that biotin targeted derivatives of 8 and 9 are still active against different cancer cell lines and that cytotoxicity is significantly increased by biotin conjugation.

Concerning the influence of the number of biotinyl residues on the cytotoxic activity, the Authors found that the addition of a second biotinyl residue does not generally result in further improvement of the potency of the biotin-ACG conjugate, with the only exception of the already mentioned biotin-squamocin conjugate 10. Indeed, this conjugate, which is characterized by two biotinyl moieties, resulted up to three times more potent and two times more selective than the corresponding mono-biotinylated derivative 12 (Figure 6). Interestingly, the Authors also noticed that the optimal site of attachment of biotin strongly depend on whether or not the 6-aminocaproic acid residue is present as a linker between the ACG scaffold and the biotinyl residue. Particularly, concerning biotin-squamocin conjugates, the preferred site of attachment of biotin is the C-28 hydroxyl group when biotin is directly attached to the scaffold, and become the C-15 hydroxyl group when the linker between biotin and squamocin is present. In the case of biotin-bullatacin conjugates, the optimal site for biotinyl derivatization is the C-15 hydroxyl group in the absence of the linker, which changes to the C-4 and C-24 hydroxyl groups in the presence of the linker. Moreover, regarding the effect of the linker on the biological activity, Authors showed that biotinACG conjugates bearing the linker are generally more potent than the analogues which lack such linker, thus proving that the presence of the linking spacer positively affect the anticancer potential of the conjugates. Indeed, the biotin-squamocin conjugate 12 (Figure 6), biotinylated at C-15 and bearing the linker between squamocin and biotin, is almost four and ten times more potent against $4 \mathrm{~T} 1$ cells and P815 cells, respectively, than the analogue without the linker. Similarly, conjugates 11 and 13 (Figure 6), biotinylated at C-24 and $\mathrm{C}-4$, respectively, and both bearing the linker between bullatacin and biotin, are twelve to forty nine times more active against $4 \mathrm{~T} 1$ cells and P815 cells, respectively, than the corresponding analogues without the linker. In summary, in this study a broad investigation of the effect of biotin conjugation on the anticancer activity of two ACGs has been described. The results clearly proved that biotin conjugation significantly increases the anticancer potential ACGs, which, in turn, is significantly affected by i) the number of biotinyl residues included in the conjugates, ii) the point of attachment of the biotinyl residue, as well as iii) the presence or not of a spacer between ACG and biotin.

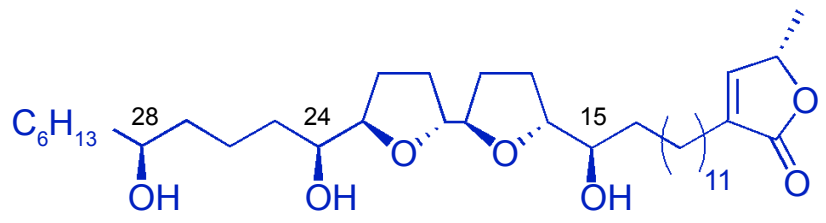

8

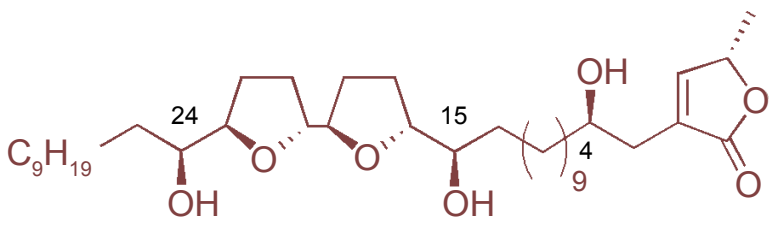

9

Figure 5: Chemical structure of Squamocin 8 and Bullatacin 9. 


\section{Biotinylation of Protein with Antitumor Effect}

\section{Biotin conjugated to $\mathrm{p} 53$ protein}

p53 is a transcription factor involved in various cellular functions, such as cell-cycle regulation, initiation of apoptotic cell death and DNA repair. Mutated or otherwise deactivated p53 is observed in the majority of human cancers [65]. Several mechanisms, i.e. mutations in p53 DNA binding domain and enhanced proteasomal degradation upon ubiquitination, are responsible for p53 inactivation. Due to its vital functions, p53 has attracted a great attention, and several approaches have been pursued to deliver p53 into tumor cells [66]. In this context, in 2013 Fahrer et al. demonstrated that biotinylation of p53 represents a successful strategy to target and deliver the protein to cancer cells [67]. In this study the Authors take advantage of the already developed C2-streptavidin transporter $[68,69]$ to introduce the biotin-p53 conjugate into various mammalian cell lines. Briefly, as reported in Figure 7, the nontoxic moiety of Clostridium botulinum C2 toxin of the recombinant C2-streptavidin fusion protein mediates the cellular uptake by a cascade of events that bring to the internalization of the toxin by a clathrin-dependent endocytosis and, on the other side, the streptavidin unit serves as a binding platform for biotin-labeled cargo molecules. Firstly, recombinant human p53 was produced in insect cells infected with p53 baculovirus [70] and then, it was properly biotinylated, yielding a biotin-p53 conjugate in which the protein was modified with 2.2 biotin groups per molecule. Next, the Authors clearly demonstrated that the biotinylation of p53 does not affect its DNAbinding activity. Indeed the DNA-binding of biotin- p53 conjugate was comparable to that of unmodified p53. Furthermore, the addition of the biotin-p53 conjugate to specific DNA sequences resulted in the formation of DNA-p53 complexes in a concentration-dependent manner. Finally, the Authors assessed the C2-streptavidin-mediated internalization of biotin-p53 conjugate in both Vero cells (monkey kidney epithelial cells) and Hela (cervix carcinoma) cells. Interestingly, an efficient internalization of biotin-p53 conjugate in a C2-streptavidindependent manner was observed in both cases. Summarizing, these data demonstrate that biotin conjugation to p53 is a successful approach to promote the C2-streptavidin mediated internalization of the transcription factor into cultured tumor cells, where the biotin-p53 conjugate resulted stable over $24 \mathrm{~h}$ and not underwent proteasomal degradation. It should be noted that the mild chemistry adopted by the Authors for the biotin conjugation to p53 could be used for wide number of labile proteins or peptides of pharmaceutical interest.

\section{Conclusions}

This review gives a highlight on recent progresses on the so-called biotin-mediated drug targeting of molecules endowed with anticancer properties. Particularly, the review has been organized in three different sections in which we have examined recent success in the conjugation of biotin with i) anticancer drugs commonly used in cancer therapy (toxoids, doxorubicin and gemcitabine) or their derivatives, as well as ii) anticancer drug candidates or iii) protein with antitumor effect, furnishing biotin conjugates able to preferentially deliver the anticancer molecules to cancer cells.

In summary, from the analysis of the literature on this topic clearly emerged that the conjugation of biotin with anticancer molecules represents an attractive and highly innovative approach to improve efficiency and efficacy and reduce cytotoxicity of anti-cancer therapy.
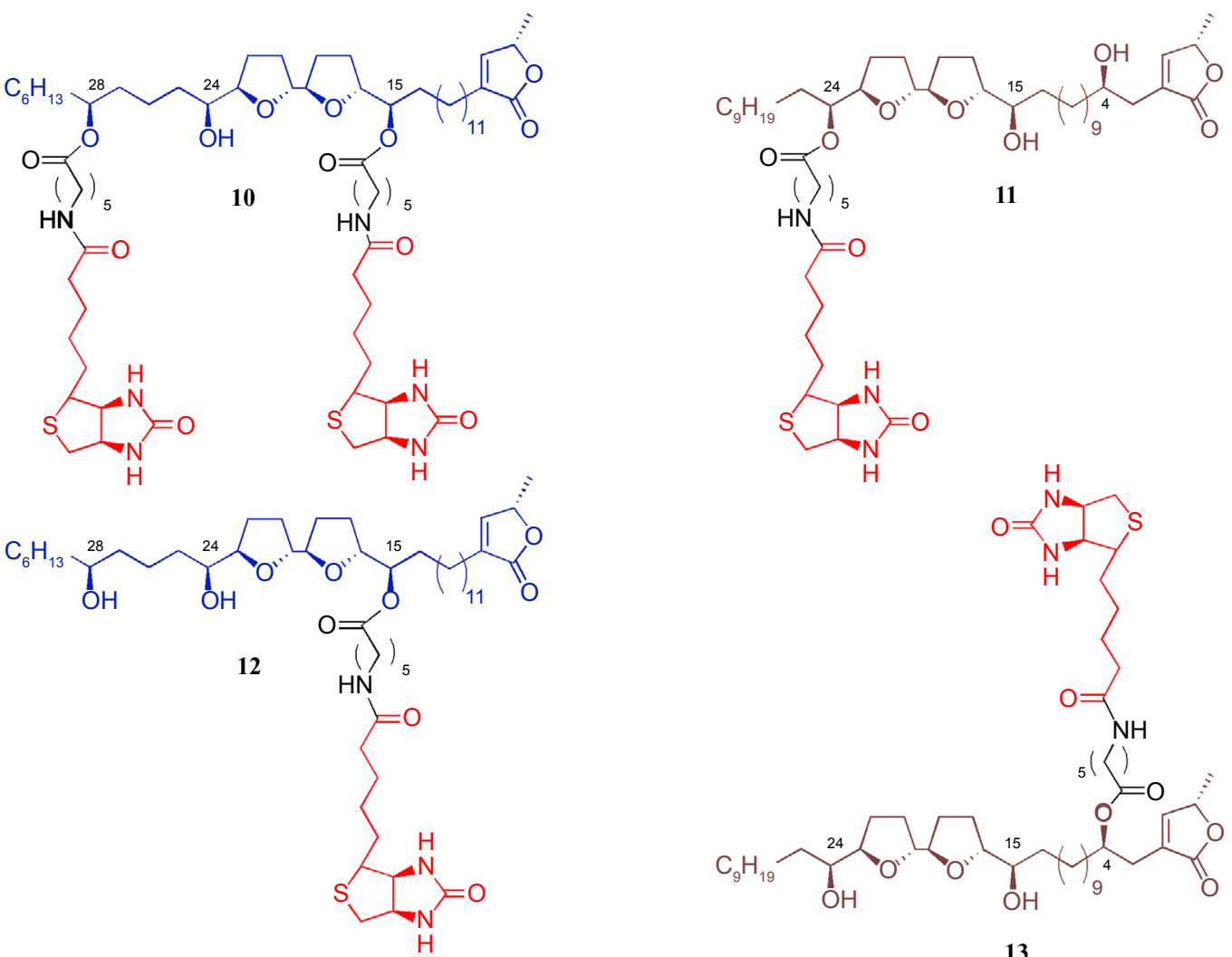

13

Figure 6: Chemical structure of biotin-squamocin conjugates 10 and 12; chemical structure of biotin- bullatacin conjugates 11 and 13. 


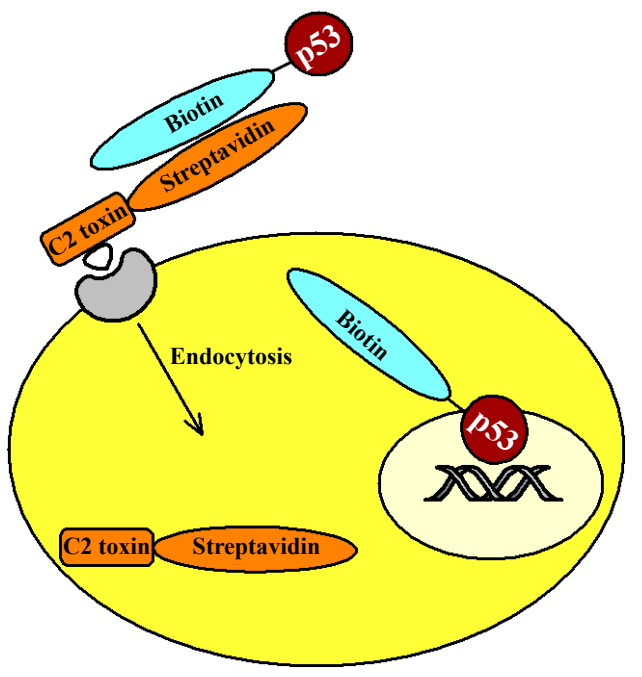

Figure 7: Schematic representation of the cellular internalization of a biotin-p53 conjugate mediated by the $\mathrm{C} 2$-streptavidin transporter.

Although only few targeted drugs have been developed so far, this area is in constant growth and we strongly believe that this approach could give an added value to cancer therapy.

\section{References}

1. Bolden JE, Peart MJ, Johnstone RW (2006) Anticancer activities of histone deacetylase inhibitors. Nat Rev Drug Discov 5: 769-784.

2. Jamieson ER, Lippard SJ (1999) Structure, Recognition, and Processing of Cisplatin-DNA Adducts. Chem Rev 99: 2467-2498.

3. Dumontet C, Jordan MA (2010) Microtubule-binding agents: a dynamic field of cancer therapeutics. Nat Rev Drug Discov 9: 790-803.

4. Jordan MA, Wilson $L$ (2004) Microtubules as a target for anticancer drugs. Nat Rev Cancer 4: 253-265.

5. Jordan MA (2002) Mechanism of action of antitumor drugs that interact with microtubules and tubulin. Curr Med Chem Anticancer Agents 2: 1-17.

6. Timmers L, Boons CC, Kropff F, van de Ven PM, Swart EL, et al. (2014) Adherence and patients' experiences with the use of oral anticancer agents. Acta Oncol 53: 259-267.

7. Mahato R, Tai W, Cheng K (2011) Prodrugs for improving tumor targetability and efficiency. Adv Drug Deliv Rev 63: 659-670.

8. Couffignal AL, Lapeyre-Mestre M, Bonhomme C, Bugat R, Montastruc JL (2000) [Adverse effects of anticancer drugs: apropos of a pharmacovigilance study at a specialized oncology institution]. Therapie 55: 635-641.

9. Mandracchia D, Tripodo G, Latrofa A, Dorati R (2014) Amphiphilic inulin-DÎt-tocopherol succinate (INVITE) bioconjugates for biomedical applications. Carbohydr Polym 103: 46-54.

10. Tripodo G, Mandracchia D, Dorati R, Latrofa A, Genta I, et al. (2013) Nanostructured Polymeric Functional Micelles for Drug Delivery Applications. Macromol Symp 334: 17-23.

11. LoPresti C, Vetri V, Ricca M, Fodera V, Tripodo G, et al. (2011) Pulsatile protein release and protection using radiation-crosslinked polypeptide hydrogel delivery devices. React Funct Polym 71: 155-167.

12. Tripodo G, Pitarresi G, Cavallaro G, Palumbo FS, Giammona G (2009) Controlled release of IgG by novel UV induced polysaccharide/poly(amino acid) hydrogels. Macromol Biosci 9: 393-401.

13. Pitarresi G, Palumbo FS, Tripodo G, Cavallaro G, Giammona G (2007) Preparation and characterization of new hydrogels based on hyaluronic acid and alpha,beta-polyaspartylhydrazide. Eur Polym J 43: 3953-3962.

14. Pitarresi G, Pierro P, Tripodo G, Mandracchia D, Giammona G (2005) Drug delivery from mucoadhesive disks based on a photo-cross-linkable polyaspartamide derivative. J Drug Deliv Sci Technol 15: 377-382.
15. Mandracchia D, Denora N, Franco M, Pitarresi G, Giammona G, et al (2010) New Biodegradable Hydrogels Based on Inulin and alpha,betaPolyaspartylhydrazide Designed for Colonic Drug Delivery: In Vitro Release of Glutathione and Oxytocin. J Biomater Sci Polym Ed.

16. Mandracchia D, Piccionello AP, Pitarresi G, Pace A, Buscemi S, et al. (2007) Fluoropolymer based on a polyaspartamide containing 1,2,4-oxadiazole units: a potential artificial oxygen (O2) carrier. Macromol Biosci 7: 836-845.

17. Giammona G, Pitarresi G, Cavallaro G, Carlisi B, Craparo EF, et al. (2006) $\mathrm{pH}$-sensitive hydrogel based on a polyaspartamide derivative. J Drug Deliv Sci Technol 16: 77-84.

18. Gibiansky L, Gibiansky E (2014) Target-mediated drug disposition model and its approximations for antibody-drug conjugates. J Pharmacokinet Pharmacodyn 41: $35-47$.

19. Lammers T, Kiessling F, Hennink WE, Storm G (2012) Drug targeting to tumors: principles, pitfalls and (pre-) clinical progress. J Control Release 161: 175-187.

20. Bareford LM, Avaritt BR, Ghandehari H, Nan A, Swaan PW (2013) Riboflavintargeted polymer conjugates for breast tumor delivery. Pharm Res 30: 17991812.

21. Nan A, Ghandehari H, Hebert C, Siavash H, Nikitakis N, et al. (2005) Watersoluble polymers for targeted drug delivery to human squamous carcinoma of head and neck. J Drug Target 13: 189-197.

22. Licciardi M, Craparo EF, Giammona G, Armes SP, Tang Y, et al. (2008) in vitro biological evaluation of folate-functionalized block copolymer micelles for selective anti-cancer drug delivery. Macromol Biosci 8: 615-626.

23. Cavallaro G, Maniscalco L, Campisi M, Schillaci D, Giammona G (2007) Synthesis, characterization and in vitro cytotoxicity studies of a macromolecular conjugate of paclitaxel bearing oxytocin as targeting moiety. Eur $\mathrm{J}$ Pharm Biopharm 66: 182-192.

24. Akdemir ZS, Akçakaya H, Kahraman MV, Ceyhan T, Kayaman-Apohan N, et al. (2008) Photopolymerized injectable RGD-modified fumarated poly(ethylene glycol) diglycidyl ether hydrogels for cell growth. Macromol Biosci 8: 852-862.

25. Axworthy DB, Reno JM, Hylarides MD, Mallett RW, Theodore LJ, et al. (2000) Cure of human carcinoma xenografts by a single dose of pretargeted yttrium-90 with negligible toxicity. Proc Natl Acad Sci U S A 97: 1802-1807.

26. Altaner C, Altanerova V, Cihova M, Ondicova K, Rychly B, et al. (2014) Complete regression of glioblastoma by mesenchymal stem cells mediated prodrug gene therapy simulating clinical therapeutic scenario. Int $\mathrm{J}$ Cancer 134: 1458-1465.

27. Elsadek B, Graeser R, Esser N, Schäfer-Obodozie C, Abu Ajaj K, et al. (2010) Development of a novel prodrug of paclitaxel that is cleaved by prostate-specific antigen: an in vitro and in vivo evaluation study. Eur J Cancer 46: 3434-3444.

28. Fortin S, Berube (2013) Advances in the development of hybrid anticancer drugs. Expert Opin Drug Discov 8: 1029-1047.

29. Jaracz S, Chen J, Kuznetsova LV, Ojima I (2005) Recent advances in tumortargeting anticancer drug conjugates. Bioorg Med Chem 13: 5043-5054.

30. Bildstein L, Dubernet C, Couvreur P (2011) Prodrug-based intracellular delivery of anticancer agents. Adv Drug Deliv Rev 63: 3-23.

31. Russell-Jones G, McTavish K, McEwan J, Rice J, Nowotnik D (2004) Vitaminmediated targeting as a potential mechanism to increase drug uptake by tumours. J Inorg Biochem 98: 1625-1633.

32. Kozyraki R, Cases O (2013) Vitamin B12 absorption: mammalian physiology and acquired and inherited disorders. Biochimie 95: 1002-1007.

33. Gupta Y, Kohli DV, Jain SK (2008) Vitamin B12-mediated transport: a potential tool for tumor targeting of antineoplastic drugs and imaging agents. Crit Rev Ther Drug Carrier Syst 25: 347-379.

34. Reddy JA, Low PS (1998) Folate-mediated targeting of therapeutic and imaging agents to cancers. Crit Rev Ther Drug Carrier Syst 15: 587-627.

35. Kolhatkar R, Lote A, Khambati $\mathrm{H}$ (2011) Active tumor targeting of nanomaterials using folic acid, transferrin and integrin receptors. Curr Drug Discov Technol 8 : 197-206.

36. Rodriguez-Melendez R, Zempleni J (2003) Regulation of gene expression by biotin (review). J Nutr Biochem 14: 680-690.

37. Zempleni J, Mock DM (1998) Uptake and metabolism of biotin by human peripheral blood mononuclear cells. Am J Physiol 275: C382-388. 
Citation: Tripodo G, Mandracchia D, Collina S, Rui M, Rossi D (2014) New Perspectives in Cancer Therapy: The Biotin-Antitumor Molecule Conjugates. Med chem S1: 004. doi:10.4172/2161-0444.S1-004

Page 8 of 8

38. Zempleni J, Mock DM (1999) Human peripheral blood mononuclear cells; Inhibition of biotin transport by reversible competition with pantothenic acid is quantitatively minor. J Nutr Biochem 10: 427-432.

39. GrassI SM (1992) Human placental brush-border membrane Na+-biotin cotransport. J Biol Chem 267: 17760-17765.

40. Chen S, Zhao X, Chen J, Chen J, Kuznetsova L, et al. (2010) Mechanism-based tumor-targeting drug delivery system. Validation of efficient vitamin receptormediated endocytosis and drug release. Bioconjug Chem 21: 979-987.

41. Shi JF, Wu P, Jiang ZH, Wei XY (2014) Synthesis and tumor cell growth inhibitory activity of biotinylated annonaceous acetogenins. Eur $\mathrm{J}$ Med Chem 71: 219-228.

42. Vadlapudi AD, Vadlapatla RK, Pal D, Mitra AK (2012) Functional and molecular aspects of biotin uptake via SMVT in human corneal epithelial (HCEC) and retinal pigment epithelial (D407) cells. AAPS J 14: 832-842.

43. Luo S, Kansara VS, Zhu X, Mandava NK, Pal D, et al (2006) Functional characterization of sodium-dependent multivitamin transporter in MDCK-MDR1 cells and its utilization as a target for drug delivery. Mol Pharm 3: 329-339.

44. Vale RD (2003) The molecular motor toolbox for intracellular transport. Cell 112: $467-480$.

45. Pitarresi G, Tripodo G, Calabrese R, Craparo EF, Licciardi M, et al. (2008) Hydrogels for potential colon drug release by thiol-ene conjugate addition of a new inulin derivative. Macromol Biosci 8: 891-902.

46. Wang Y, O'Brate A, Zhou W, Giannakakou P (2005) Resistance to microtubulestabilizing drugs involves two events: beta-tubulin mutation in one allele followed by loss of the second allele. Cell Cycle 4: 1847-1853

47. Lis LG, Smart MA, Luchniak A, Gupta ML Jr, Gurvich VJ (2012) Synthesis and Biological Evaluation of a Biotinylated Paclitaxel With an Extra-Long Chain Spacer Arm. ACS Med Chem Lett 3: 745-748.

48. Nicolaou KC, Dai WM, Guy RK (1994) Chemistry and Biology of Taxol. Angew Chem-Int Edit 33: 15-44.

49. Gewirtz DA (1999) A critical evaluation of the mechanisms of action proposed for the antitumor effects of the anthracycline antibiotics adriamycin and daunorubicin. Biochem Pharmacol 57: 727-741.

50. Minotti G, Menna P, Salvatorelli E, Cairo G, Gianni L (2004) Anthracyclines: molecular advances and pharmacologic developments in antitumor activity and cardiotoxicity. Pharmacol Rev 56: 185-229.

51. Ibsen S, Zahavy E, Wrasdilo W, Berns M, Chan M, et al. (2010) A novel Doxorubicin prodrug with controllable photolysis activation for cancer chemotherapy. Pharm Res 27: 1848-1860.

52. Sutherland JC, Griffin KP (1981) Absorption spectrum of DNA for wavelengths greater than $300 \mathrm{~nm}$. Radiat Res 86: 399-409.

53. Weiden PL, Breitz HB (2001) Pretargeted radioimmunotherapy (PRIT) for treatment of non-Hodgkin's lymphoma (NHL). Crit Rev Oncol Hematol 40: $37-$ 51.

54. Friberg G, Kindler HL (2005) Chemotherapy for advanced pancreatic cancer: past, present, and future. Curr Oncol Rep 7: 186-195.
55. Maiti S, Park N, Han JH, Jeon HM, Lee JH, et al. (2013) Gemcitabine-coumarinbiotin conjugates: a target specific theranostic anticancer prodrug. J Am Chem Soc 135: 4567-4572.

56. Mini E, Nobili S, Caciagli B, Landini I, Mazzei T (2006) Cellular pharmacology of gemcitabine. Ann Oncol 17 Suppl 5: v7-12.

57. Noble S, Goa KL (1997) Gemcitabine. A review of its pharmacology and clinical potential in non-small cell lung cancer and pancreatic cancer. Drugs 54: 447472.

58. Bhuniya S, Lee MH, Jeon HM, Han JH, Lee JH, et al. (2013) A fluorescence offon reporter for real time monitoring of gemcitabine delivery to the cancer cells. Chem Commun (Camb) 49: 7141-7143.

59. Lee MH, Kim JY, Han JH, Bhuniya S, Sessler JL, et al. (2012) Direct fluorescence monitoring of the delivery and cellular uptake of a cancer-targeted RGD peptide-appended naphthalimide theragnostic prodrug. J Am Chem Soc 134: $12668-12674$.

60. Degli Esposti M, Ghelli A, Ratta M, Cortes D, Estornell E (1994) Natural substances (acetogenins) from the family Annonaceae are powerful inhibitors of mitochondrial NADH dehydrogenase (Complex I). Biochem J 301: 161-167.

61. Londershausen M, Leicht W, Lieb F, Moeschler H, Weiss H (1991) Molecularmode of action of annonins. Pestic Sci 33: 427-438.

62. Duval R, Lewin G, Hocquemiller R (2003) Semisynthesis of heterocyclic analogues of squamocin, a cytotoxic annonaceous acetogenin, by an unusual oxidative decarboxylation reaction. Bioorg Med Chem 11: 3439-3446.

63. Derbre S, Roue G, Poupon E, Susin SA, Hocquemiller R (2005) Annonaceous acetogenins: The hydroxyl groups and THF rings are crucial structural elements for targeting the mitochondria, demonstration with the synthesis of fluorescent squamocin analogues. Chem Bio Chem 6: 979-982.

64. McLaughlin JL (2008) Paw paw and cancer: annonaceous acetogenins from discovery to commercial products. J Nat Prod 71: 1311-1321.

65. Romer L, Klein C, Dehner A, Kessler H, Buchner J (2006) p53--a natural cancer killer: structural insights and therapeutic concepts. Angew Chem Int Ed Engl 45: 6440-6460.

66. Horn HF, Vousden $\mathrm{KH}$ (2007) Coping with stress: multiple ways to activate p53 Oncogene 26: 1306-1316.

67. Fahrer J, Schweitzer B, Fiedler K, Langer T, Gierschik P, et al. (2013) C2Streptavidin Mediates the Delivery of Biotin-Conjugated Tumor Suppressor Protein p53 into Tumor Cells. Bioconjugate Chem 24: 595-603.

68. Fahrer J, Plunien R, Binder U, Langer T, Seliger H, et al. (2010) Genetically engineered clostridial $\mathrm{C} 2$ toxin as a novel delivery system for living mammalian cells. Bioconjug Chem 21: 130-139.

69. Fahrer J, Rieger J, van Zandbergen G, Barth H (2010) The C2-streptavidin delivery system promotes the uptake of biotinylated molecules in macrophages and T-leukemia cells. Biol Chem 391: 1315-1325.

70. Fahrer J, Kranaster R, Altmeyer M, Marx A, Burkle A (2007) Quantitative analysis of the binding affinity of poly(ADP-ribose) to specific binding proteins as a function of chain length. Nucleic Acids Res 35: e143.
This article was originally published in a special issue, Cancer Prevention and Therapy handled by Editor(s). Dr. Dong Xiao, University of Pittsburgh School of Medicine, USA 